\title{
Transcatheter occlusion of coronary to bronchial anastomosis by detachable balloon combined with coronary angioplasty at same procedure
}

\author{
J F REIDY, E SOWTON, D N ROSS
}

From the Departments of Radiology and Cardiology, Guy's Hospital, London; and Harley Street Clinic, London

SUMMARY The first case of non-operative occlusion of a large coronary to bronchial anastomosis is? described. The patient who had:severe angina had also a critical stenosis of the anterior descending 3 coronary artery which was successfully dilated by an angioplasty procedure. An occluding balloon $\frac{\vec{n}}{3}$ was detached in the large distal circumflex coronary artery, beyond all the normal branches. At $a^{\stackrel{\rho}{乛}}$ repeat catheterisation study seven months later the balloon was intact and in position, and the $\vec{\infty}$ anastomosis remained occluded.

There have been many descriptions of coronary artery to bronchial artery anastomosis published. ${ }^{1-3}$ Small anastomoses, presumably congenital in origin, have been noted to occur in about $20 \%$ of normal subjects in the absence of other abnormalities. ${ }^{1}$ Larger anastomoses have been seen in one of two situations: when there is significant coronary artery disease the bronchial arteries may communicate via collaterals with the coronary artery distal to the site of obstruction. ${ }^{1}$ The opposite situation occurs when there is severe right ventricular outflow tract obstruction and then the coronary to bronchial anastomoses communicate with the lower pressure pulmonary circulation. In both these situations the coronary to bronchial anastomoses are of secondary significance compared with the primary disease and no specific treatment is usually needed. In the case described here there was a large haemodynamically significant coronary to bronchial anastomosis that occurred as an isolated finding and not secondary to any other abnormality.

Much has been written on congenital coronary artery fistulae and in the majority of cases surgical correction has been advocated. ${ }^{4}$

We present a case of a patient with severe angina who was successfully treated by the procedure of combined coronary angioplasty and transcatheter occlusion of a large haemodynamically significant coronary artery to bronchial artery anastomosis. To our knowledge this is the first case of its kind to be described and this now offers a new form of treatment for these uncommon but significant anastomoses.

\section{Case report}

The patient is a man of 67 who was referred by $\mathrm{D} \frac{\mathrm{D}}{\mathrm{D}}$ Palencia Perez in Valencia for possible surgical treat $\varrho$ ment. His history was of admission to hospital with $\overrightarrow{\vec{b}}$ acute coronary insufficiency including unstable ST3 segment elevation on the electrocardiogram, wide? spread $T$ wave changes suggesting anterior suben: docardial infarction, and repeated episodes of pain He was treated with bed rest, isosorbide, nifedipine and metoprolol, and in between the episodes of pair his electrocardiogram always returned to normal. The symptoms settled without any evidence of actuab infarction and coronary arteriography was subse quently performed in Spain. This showed a severeo stenosis of the proximal left anterior descending coro nary artery and a large circumflex coronary artery to bronchial anastomosis. There was no clinical evidence of respiratory disease.

Clinical examination was normal. There were no detectable murmurs; electrocardiogram and chest $x$-ray film were normal.

In view of the patient's age and the type of lesions if was decided to attempt a coronary angioplasty anc embolisation of the circumflex anastomosis at the same procedure with full surgical standby in case of complications.

In May 1981 cardiac catheterisation was performe via the right percutaneous femoral approach. Thi again showed severe proximal stenosis of the anteriog descending artery $(>90 \%)$ but with no other occlusive 


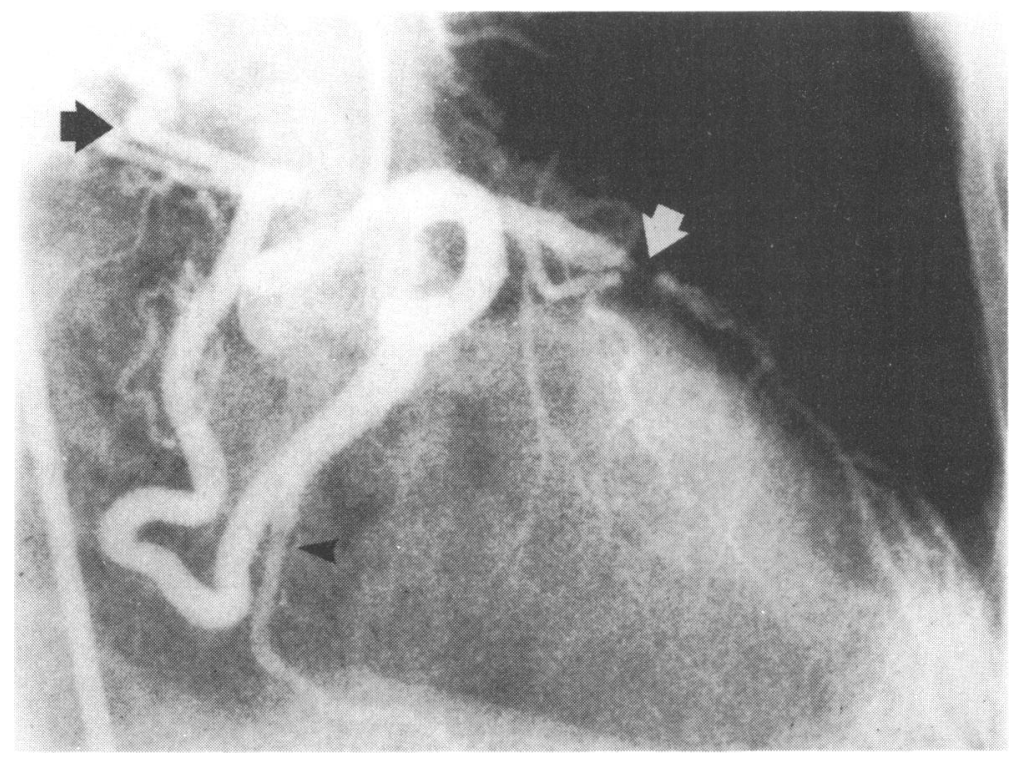

Fig. 1 Left coronary arteriogram in the right anterior oblique projection. There is a severe proximal stenosis of the anterior descending branch (white arrow). Note the very large calibre of the circumflex artery, the last obtuse marginal branch (black arrowhead), and the distal artery just before it runs into the bronchial anastomoses (black arrow).

disease. The circumflex artery was of very large calibre. After it gave off a distal obtuse marginal branch it continued as a large and tortuous artery behind the heart (Fig. 1). Passing in a superior direction it terminated in a leash of small vessels situated in the region of the left hilum and the bronchial arteries (Fig. 2). There was no evidence of any communication with pulmonary arteries. Left ventricular angiography and pressure studies were all normal.

Both the angioplasty and the embolisation procedures ware performed via a 9F non-tapered coronary guide catheter (Schneider Medintag Ag). The angioplasty was performed first and a gradient of 105 $\mathrm{mmHg}$ was abolished with a $3.7 \mathrm{~mm}$ diameter dilatation catheter.

The same introducer catheter was then manipulated into the proximal circumflex artery and the embolisation achieved using the Becton-Dickinson detachable balloon catheter. ${ }^{\star}$ When safely positioned in the distal circumflex coronary artery the balloon was inflated with contrast medium while a test injection was made through the coronary guide catheter. When this confirmed both a good position of the balloon and showed that it was occluding the artery, it was detached by gentle traction. The silicone balloon was inflated with iso-osmotic contrast medium. There

^Becton-Dickinson, Rutherford, New Jersey.

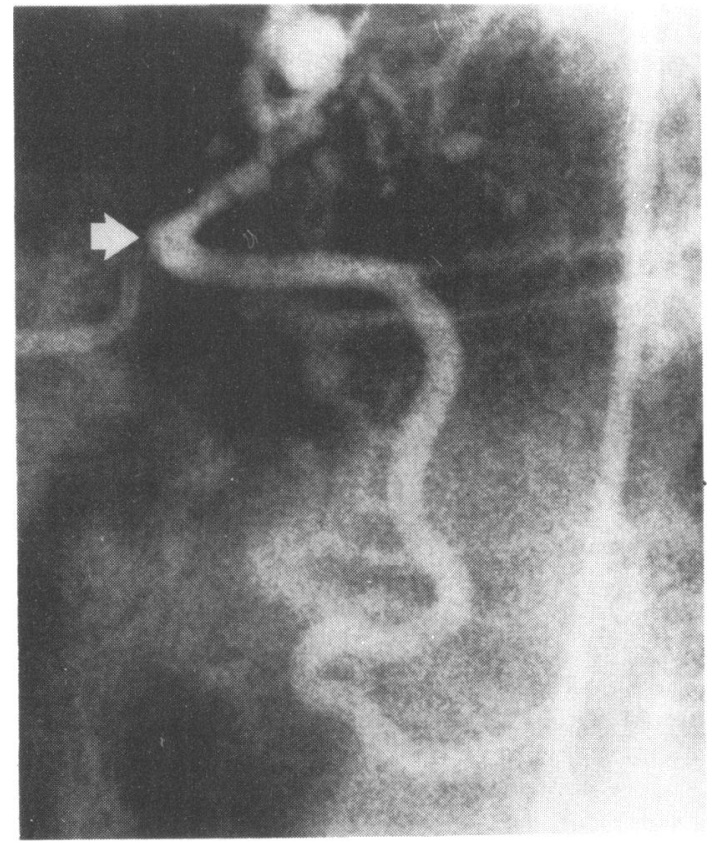

Fig. 2 Left coronary arteriogram in the left anterior oblique projection showing the most distal part of the circumflex coronary artery where it runs to join the bronchial arteries. Note the most terminal part (white arrow) that corresponds with the black arrow of Fig 1. 


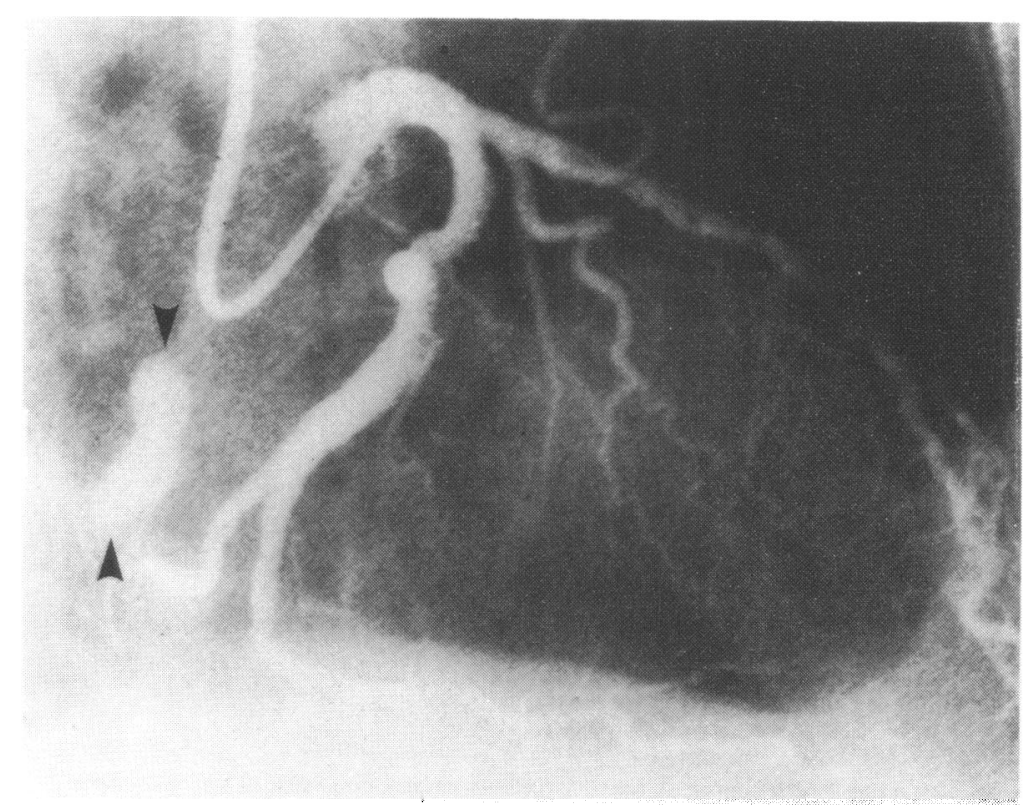

Fig. 3 Left coronary arteriogram seven months after coromary embolisation and angioplasty. There is a good angioplasty result. The anastomosis remains occluded and the balloon, still filled with contrast (between black amowheads), is well distal to the last obtuse manginal branch (cf Fig. 1).

were no complications and the patient was discharged from hospital four days later.

The patient was seen at follow up seven months after the angioplasty and embolisation. He has been completely free of symptoms and able to take vigorous exercise. In December 1981 a follow up sardiac catheterisation was performed. The left coronary arteriogram showed a little irregularity at the site of the anterior descending angioplasty but no stenosis (Fig. 3). The balloon was still intact and completely occluding the distal circumflex artery and fistula. The patient remains symptom free in August 1982, 15 months after the procedure.

\section{Discucsion}

The finst case of a coronary to bronchial anastomosis demonstrated on selective coronary arteriography was in 1972 in a patient who had normal coronary arteries but had evidence of obstructive airways disease. ${ }^{3}$ Two patients shown at coronary arteriography to have large coronary to bronchial anastomoses were also shown to have no coronary artery disease, but both of these patients had bronchiectasis as indicated by bronchography. ${ }^{2}$ One of these patients had severe angina associated with electrocardiographic changes and was treated by lower lobectomy combined with ligation of the anastomotic vessel.
The other patient who had no history of angina and only mild respiratory symptoms was managed by con윽 servative treatment.

Our patient presented with severe angina but hat no evidence of respiratory disease. In addition to the large coronary to bronchial anastomosis arising fron the circumflex artery, there was a severe and critical stenosis of the proximal anterior descending coronary? artery. The remaining coronary arteries were normal The bronchials filled from the left coronary injection and there was no evidence of collateral vessels between the circumflex artery and the distal anterio descending to suggest that there was a bronchial tof coronary "steal". In the absence of any evidence of. respiratory disease, the coronary to bronchial anas 0 tomosis appears to be a primary and presumably congenital anomaly.

The very large size of the circumflex coronary artery and the distal branch passing to the bronchia artery territory suggest that this anastomosis was of functional significance.

Thus our case had two clear-cut abnormalities that could cause his angina. As the proximal anterio descending stenosis was very suitable for an angio plasty procedure, it was decided to acclude the large coronary to bronchial anastomosis with a detachable balloon at the same occasion and via the same cathete approach and introducing catheter. Of all the technio 
ques available for occluding vessels, the detachable balloon was the only one that was suitable for this situation.

Detachable balloons have been used in many vessels but only recently in the cardiopulmonary situaion. ${ }^{5}$ As the large branch arose distal to the origin of all the obtuse marginal branches of the circumflex, it was essential to occlude the vessel distal to these. Passing a catheter down into the distal circumflex presents many problems but using the method whereby the small uninflated balloon could be injected out along with the forward blood flow proved an ideal method. The balloon was filled with iso-osmotic contrast media, and there is evidence that if a vessel can be occluded for a minimum of 10 days then the vessel will remain occluded. To our knowledge this is the first reported case of deliberate occlusion of a coronary artery vascular lesion via a percutaneous transcatheter approach.

We are grateful to $\mathrm{Dr}$ RI White for advice and encouragement that enabled us to start using the detachable balloon occlusion system.

\section{References}

1 Björk L. Angiographic demonstration of extra-cardial anastomoses to the coronary arteries. Radiology 1966; 87: $274-7$.

2 Sutton NG StJ, Miller GAH, Kerr IH, Traill TA. Coronary artery steal via a large coronary artery to bronchial artery anastomosis successfully treated by operation. $\mathrm{Br}$ Heart f 1980; 44: 460-3.

3 Smith SC, Adams DF, Herman MV, Paulin S. Coronary-to-bronchial anastomoses: an in vivo demonstration by selective coronary arteriography. Radiology 1972; 104: 289-90.

4 Levin DC, Fellows KE, Abrams HL. Hemodynamically significant primary anomalies of the coronary arteries. Circulation 1978; 58: 25-34.

5 Grinnell VS, Mehringer CM, Hieshima GB, Stanley P, Lurie PR. Transaortic occlusion of collateral arteries of the lung by detachable valved balloons in a patient with tetralogy of Fallot. Circulation 1982; 65: 1276-8.

Requests for reprints to Dr John F Reidy, X-Ray Department, Guy's Hospital, St Thomas Street, London SE1 9RT. 